$\mathrm{DOE} / \mathrm{ER} / 40286--\mathrm{T} 4$

DE92 003038

\title{
CONFIGURATION SPACE FADDEEV CALCULATIONS
}

\section{Progress Report}

for Period 1 January 1988 - 31 December 1988

G. L. PAYNE, W. H. KLINK, and W. N. POLYZOU

Department of Physics and Astronomy

The University of Iowa

Iowa City, Iowa 52242-1479

January 1989

Prepared for

THE U. S. DEPARTMENT OF ENERGY

AGREEMENT NO. DE-FG02-86ER40286 
TABLE OF CONTENTS

Page

ABSTRACT ........................ . $\mathrm{jii}$

I. PERSONNEL . . . . . . . . . . . . . . . . . . . . . 1

Principal Investigators . . . . . . . . . . . . . . . . . . . . . 1

Graduate Student Research Assistants . . . . . . . . . . . . . . 1

Time Devoted to Contract . . . . . . . . . . . . . . . . . . . 1

II. SUMMARY OF RESEARCH COMPLETED . . . . . . . . . . . 2

Trinucleon Asymptotic Normalization Constants:

A Comparison of ${ }^{3} \mathrm{He}$ and ${ }^{3} \mathrm{H}$. . . . . . . . . . . . . . . . 2

${ }^{3} \mathrm{H}$ and ${ }^{3} \mathrm{He}$ Solutions for Momentum-Dependent Potentials . . . . . . . 3

S-Wave $\pi \mathrm{N}$ Scattering Effects in the Nuclear Three-Body Force . . . 4

Triton Model Calculation Test of the Bonn W-Matrix

Rank-One Separable Approximation . . . . . . . . . . . . . 5

Low-Energy Nucleon-Deuteron Scattering . . . . . . . . . . . . . 6

Spectral Transform for the Sub-Laplacian on the Heisenberg Group . . 8

On Resolving the Multiplicity of Arbitrary Tensor Products of the

$U(N)$ Groups . . . . . . . . . . . . . . . . . . . . 9

Scattering Operators on Fock Space

IV. The Algebra of Operators Commuting with an Internal Symmetry

V. The Pseudoscalar Mesons . . . . . . . . . . . . . . . . . . 9

Elastic Electron-Deuteron Scattering . . . . . . . . . . . . . . . 10

Tensor Operators in Poincaré Invariant Models . . . . . . . . . . . 11

Charge Form Factor of Quark-Model Pions . . . . . . . . . . . . 12

Analyzing Multiparticle Reactions I, II . . . . . . . . . . . . . . 13

Relativistic Two-Body Models . . . . . . . . . . . . . . . . . 13

III. PAPERS PUBLISHED, SUBMITTED OR ACCEPTED . . . . . . 14

Papers Published (1 January 1988 - 31 December 1988) . . . . . . 15

Papers Submitted or Accepted (as of 31 December 1988) . . . . . . 16

Meetings Attended . . . . . . . . . . . . . . . . . . . 18

Invited Talks . . . . . . . . . . . . . . . . . . . . . 18

\section{DISCLAIMER}

This report was prepared as an account of work sponsored by an agency of the United States Government. Neither the United States Jovernment nor any agency thereof, nor any of their employees, makes any harranty, express or implied, or assumes any legal liability or responsibility for the accuracy, completeness, or usefulness of any information, apparatus. product. or ficcess disclosed, or represents that its use Hould not infringe privately ouned rights. Reference herein to any specific commercial prodict, process, or service by trade name, trademark, manufacturer, or otherwise does not neces urily constitute or imply its endorsement. recommendation, or favoring by the United States Government or any agency thereof. The views and opinions of authors expressed herein do not necessarily state or reflect those of the United States Government or any agency thereot. 


\begin{abstract}
The detailed study of few-body systems provides one of the most effective means for studying nuclear physics at subnucleon distance scales. For few-body systems the model equations can be solved numerically with errors less than the experimental uncertainties. We have used such systems to investigate the size of relativistic effects, the role of meson-exchange currents, and the importance of quark degrees of freedom in the nucleus. Complete calculations for momentum-dependent potentials have been performed, and the properties of the three-body bound state for these potentials have been studied. Few-body calculations of the electromagnetic form factors of the deuteron and pion have been carried out using a front-form formulation of relativistic quantum mechanics. The decomposition of the operators transforming covariantly under the Poincaré group into kinematical and dynamical parts has been studied. New ways for constructing interactions between particles, as well as interactions which lead to the production of particles, have been constructed in the context of a relativistic quantum mechanics. To compute scattering amplitudes in a nonperturbative way, classes of operators have been generated out of which the phase operator may be constructed. Finally, we have worked out procedures for computing Clebsch-Gordan and Racah coefficients on a computer, as well as giving procedures for dealing with the multiplicity problem.
\end{abstract}




\section{PERSONNEL}

\section{Principal Investigators}

Gerald L. Payne, Professor of Physics Department of Physics and Astronomy The University of Iowa Iowa City, Iowa 52242-1479

Telephone: (319)335-1854

William H. Klink, Professor of Physics Department of Physics and Astronomy The University of Iowa Iowa City, Iowa 52242-1479

Telephone: (319)335-1757

Wayne N. Polyzou, Associate Professor of Physics Department of Physics and Astronomy The University of Iowa Iowa City, Iowa 52242-1479

Telephone: (319)335-1856

\section{Graduate Student Research Assistants}

M. Rogers

H. Jean

\section{Time Devoted to Contract}

Gerald L. Payne

William H. Klink

Wayne N. Polyzou
$40 \%$

$40 \%$

$60 \%$

On the following pages is a summary of the theoretical research which has been carried out under Contract No. DE-FG02-86ER40286 at the Department of Physics and Astronomy of the University of Iowa. The requirements of the contract have been fulfilled. 


\title{
II. SUMMARY OF RESEARCH COMPLETED
}

\section{Trinucleon Asymptotic Normalization Constants: \\ A Comparison of ${ }^{3} \mathrm{He}$ and ${ }^{3} \mathrm{H}$}

\author{
J. L. Friar, B. F. Gibson, D. R. Lehman, and G. L. Payne
}

Our previous calculations of the trinucleon S- and D-wave asymptotic normalization constants [1], with and without Coulomb effects, were extended to include two-body partial waves up to $j \leq 4$ (34 three-body channels) [2]. The asymptotic normalization constant reflects the internal dynamics of the three-body wave function through the overall normalization, and the S-wave asymptotic normalization constant is defined such that its value is unity when the effer+ive nuclear interaction in the amptotic channel of interest is a zero-range interaction. This definition applies whether a Coulomb interaction is present or not. Wave functions were generated with our configuration-space Faddeev code for Hamiltonians based on the two-body potentials of Reid and the Argonne group. In addition, we used model Hamiltonians which included a three-body force to generate wave functions for both the Tucson-Melbourne and the Brazilian models. The wave functions were then used to calculate the constants $C_{S}$ and $C_{D}$ for ${ }^{3} \mathrm{He}$ and ${ }^{3} \mathrm{H}$ for the various model Hamiltonians.

Sinre the model Hamiltonians had a range of values for the trinucleon bound-state energy, we plotted the values of $C_{S}$ and $C_{D}$ as a function of the binding energy and found a strong correlation of the values with the binding energy. Using a least-squares fit to this plot, we estimated the best values for the asymptotic normalization constants. We found $C_{S}=1.850$ and $C_{D}=0.08 .5$ for ${ }^{3} \mathrm{H}$, and $C_{S}=1.8 .54$ and $C_{D}=0.080$ for ${ }^{3} \mathrm{He}$. 
Our results were in reasonable agreement with the existing experimental data; however, improved experimental precision is needed in order to fully test the predictions.

[1] J. L. Friar, B. F. Gibson, D. R. Lehman, and G. L. Payne, Phys. Rev. C 25 (1982), 1616.

[2] J. L. Friar, B. F. Gibson, D. R. Lehman, and G. L. Payne, Phys. Rev. C 37 (1988), 2589.

\section{${ }^{3} \mathrm{H}$ and ${ }^{3} \mathrm{He}$ Solutions for Momentum-Dependent Potentials}

J. L. Friar, B. F. Gibson, and G. L. Payne

We have modified our bound-state configuration-space Faddeev codes to include the momentum-dependent interactions such as the Paris, Nijmegen, or Bonn two-body potentials. Since we use the configuration-space approach, we could easily include a Coulomb potential to model ${ }^{3} \mathrm{He}$ as well as ${ }^{3} \mathrm{H}$. Using this code, we solved the configuration-space Faddeev equations for model Hamiltonians of the three potentials listed above [1]. Our triton binding energies agreed with previous results for the Paris and Bonn potentials, and our new Nijmegen solution had a binding energy commensurate with the other realistic two-body interactions. Using the wave functions obtained from the Faddeev calculations, we calculated the rms charge radius, the asymptotic normalization constants, and the charge density for ${ }^{3} \mathrm{H}$. Adding the Coulomb potential to the model Hamiltonian, we repeated our calculations for ${ }^{3} \mathrm{He}$. In addition, we calculated the Coulomb energies for the various models. Most of the calculated observables for the two trinucleon systems agreed well with our previous calculations for other realistic two-body potentials.

[1] J. L. Friar, B. F. Gibson, and G. L. Payne, Phys. Rev. C 37 (1988), 2869. 


\title{
S-Wave $\pi N$ Scattering Effects in the Nuclear Three-Body Force
}

\author{
J. L. Friar, B. F. Gibson, G. L. Payne, and S. A. Coon
}

A detailed investigation of the contributions of the off-mass-shell, s-wave $\pi \mathrm{N}$ scattering amplitude terms in the Tucson-Melbourne two-pion-exchange three-body force to the triton binding energy was performed [1]. Because of the sensitivity of the triton binding energy to the short-range properties of the model, the three-body force depends upon the short-range repulsion of the nucleon-nucleon interaction. Two nucleon force models with different short-range characterisitics were used in this study. The Reid soft-core two-body potential was chosen as an example of an interaction with a strong repulsive core, and the Argonne V14 was chosen as an example of a soft-core potential. Using these two potentials with the full Tucson-Melbourne three-body potential, we solved the configuration-space Faddeev equations for the triton binding energy. Then the calculations were repeated with the a and $c$ terms of the Tucson-Melbourne potential set equal to zero. It is the a and $\mathrm{c}$ terms in the potential which are derived from the s-wave part of the $\pi \mathrm{N}$ amplitude. For the Reid potential, it was found that turning off the a and $\mathrm{c}$ terms in the three-body potential only lowered the binding energy by $160 \mathrm{keV}$. Thus, perturbation theory should be more than adequate for treating the case with the Reid potential. However, for the Argonne V14 potential, turning off the a and $c$ terms results in a change for the binding energy of $600 \mathrm{keV}$. Therefore, for the Argonne V14 potential, which has a weaker short-range repulsion, the s-wave components of the three-body force produce effects that are nonperturbative in nature. Since the $c$ term in the Tucson-Melbourne three-body potential can produce sizable nonperturbative effects in the trinucleon system, a careful treatment of the full two-pion-exchange three-nucleon force is required before nhenomenological short-range terms can be investigated. 
[1] J. L. Friar, B. F. Gibson, G. L. Payne, and S. A. Coon, Few-Body Systems 5 (1988), 13.

\section{Triton Model Calculation Test of the Bonn W-Matrix Rank-One Separable Approximation}

B. F. Gibson, B. C. Pearce, and G. L. Payne

Recently it has been suggested that the off-shell t-matrix in the three-body momentum space calculculations could be approximated by the single separable term in the W-matrix form of the t-matrix [1]. That is, it was suggested that the remainder term in the $\mathrm{W}$-matrix representation of the two-body t-matrix might make a negligible contribution to the numerical calculation. This conjecture was based on the good results which were obtained for the triton bound-state energy found by using the the rank-one approximation to the Malfliet-Tjon I-III potential. This approximation yielded a binding energy of $8.59 \mathrm{MeV}$, which is very close to the result of $8.58 \mathrm{MeV}$ found in the complete calculation.

As a test of the above conjecture, we applied the $\mathrm{W}$-matrix prescription to a more realistic two-body potential [2] (one possessing a strong short-range repulsion). One case which was investigated was the a simple model based on the Reid spin-singlet interaction. The strength of this interaction was increased to produce a three-body bound state with a binding energy of approximately $7 \mathrm{MeV}$. Using this modified interaction in our configuration-space bound state code, we found a binding energy of $7.1 \mathrm{MeV}$, while the W-matrix approximation yielded a binding energy of $9.1 \mathrm{MeV}$. Apparently, neglecting the nonseparable term in the W-matrix equation is not a good approximation for this case. Thus, using only the leading separable term of the W-matrix representation of the t-matrix is not justified for all two-body potentials.

[1] E. A. Bartnik, H. Haberzettl, and W. Sandhas, Phys. Rev. C 34 (1986), 1520. 
[2] B. F. Gibson, B. C. Pearce, and G. L. Payne, submitted to Phys. Rev. C.

\title{
Low-Energy Nucleon-Deuteron Scattering
}

\author{
C. R. Chen, G. L. Payne, J. L. Friar, and B. F. Gibson
}

Several years ago, we solved [1] the three-body Faddeev equations in configuration space for the zero-energy scattering problem, and then we used the wave functions found in these calculations to determine the $\mathrm{n}-\mathrm{d}$ and $\mathrm{p}$-d scattering lengths. Calculations for various realistic two-body potentials gave results for the $n$-d scattering length in good agreement with the experimental values [2]. However, the p-d doublet scattering length from these calculations did not agree with the experimental value $[3,4]$ nor with other theoretical calculations [5]. One possibility for this difference was that the procedure used in extrapolating the experimental results at finite energies to zero energy might be incorrect. This would be true if there were a pole in the scattering amplitude at low energies. In order to test this possibility, we modified our zero-energy scattering code to include the cases for energies below the three-body breakup threshold. Using the revised code, we generated a set of numerical phase shifts which could then be used to determine the scattering length in the same manner as used for the experimental data [6]. Therefore, using our theoretical data set, we calculated the Coulomb modified effective-range function

$$
K(E)=C_{0}^{2}(\eta) k \cot \delta_{0}(k)+2 k \eta h(\eta)
$$

where $C_{0}(\eta)$ is the Coulomb penetration factor [7], $k$ is the elastic scattering wave number, and $\eta$ is the Coulomb parameter for $\mathrm{p}-\mathrm{d}$ scattering. The function $h(\eta)$ is defined as

$$
h(\eta)=-\ln \eta+\operatorname{Re} \psi(1+i \eta)
$$


and $\psi$ is the Digamma function [7]. The values of $K(E)$ were then fit by the approximation

$$
K(E) \approx \frac{-\frac{1}{a}+\frac{1}{2} r_{0} k^{2}}{1+\frac{k^{2}}{k_{0}^{2}}}
$$

Using this fit to the numerically generated data, we then determined the value of the scattering length, $a$, and the position of the pole due to a virtual state. The extrapolated value for the doublet scattering length is ${ }^{2} a_{p d}=0.17 \mathrm{fm}$ which is in excellent agreement with our value determined from the zero-energy scattering equation. In addition, we found that our crlculated values were in agreement with the experimental data.

We found that the $\mathrm{p}$-d effective-range function has a pole very near to the origin. Using the value of $k_{0}^{2}$ obtained from our fit using Equation (3), we determined that this pole is located at $E \approx-25 \mathrm{keV}$. Clearly, the experimental data does not extend to low enough values of the energy to detect the presence of this pole. Accurate experiments for energies below $300 \mathrm{keV}$ are necessary to obtain the doublet scattering length. The difference between our zero-energy results and the numerical calculaticns by the Leningrad group [5] is also due the presence of this pole. The finite-energy calculations in Reference [5] were done for energies above $500 \mathrm{keV}$ and the extrapolation was done using these phase shifts. Thus, these calculations did not go to low enough energies to detect the presence of the pole.

[1] J. L. Friar, B. F. Gibson, G. L. Payne, and C. R. Chen, Phys. Rev. C 30 (1984), 1121.

[2] W. Dilg, L. Koester, and W. Nistler, Phys. Lett. 36B (1971), 208.

[3] J. Arvieux, Nucl. Phys. A221 (1974), 253.

[4] E. Hutte' W. Arnold, H. Baumgart, H. Berg, and G. Clausnitzer, Nucl. Phys. A406 $(1983),+43$.

[5] A. A. Kivitsinskii, JETP Lett. 36 (1982), 455.

[6] C. R. Chen, G. L. Payne, J. L. Friar, and B. F. Gibson, submitted to Phys. Rev. C. 


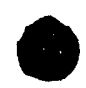

[7] M. Abramowitz and I. A. Stegun, Handbook of Mathematical Functions (U.S. GPO, Washington, DC, 1972), p. 258.

\section{Spectral Transform for the Sub-Laplacian on the Heisenberg Group [1]}

\section{P.E.T. Jorgensen and W. H. Klink}

This paper is the second in a series which attempts to compute the time-dependent Green's function for systems that have polynomial interactions. We have previously shown [2] that all systems with polynomial interactions, such as the anharmonic oscillators and systems in external (polynomial) electromagnetic fields are related to nilpotent groups. In fact the Hilbert space which describes a physical system is a representation space of a nilpotent group. This in turn means that the Hamiltonian can be written as a quadratic sum of Lie algebra elements of the underlying nilpotent group, so that the time-dependent Green's function is related to the so-called "heat kernel" of the nilpotent group.

The point of this paper is to demonstrate a technique for solving the heat equation for the Heisenberg group which is the nilpotent group related to a particle in a constant magnetic field. The quantum mechanical behavior of a particle in a constant magnetic field is of course known, but in this paper we show how to find the time-dependent Green's function by using new techniques that we hope will prove effective for quantum mechanical systems such as the anharmonic oscillator, where the solutions to the Schrödinger equation are not known.

[1] P.E.T. Jorgensen and W. H. Klink, Journal d'Analyse Mathématique 50 (1988), 101.

[2] P.E.T. Jorgensen and W. H. Klink, Publ. RIMS, 21 (1985), 969. 


\section{On Resolving the Multiplicity of Arbitrary Tensor Products of the $U(N)$ Groups}

W. H. Klink and T. Ton-That

A longstanding problem in the representation theory of compact groups, arising out of the applications of group theory to nuclear spectroscopy, is the multiplicity problem. When a representation of a group is decomposed into the irreducible representations of a subgroup, or when a tensor product of representations of a group is decomposed into irreducible representations, a given irreducible representation may appear more than once in the decomposition. How to break the degeneracy of identical spaces is the multiplicity problem.

In this paper we follow a method initiated by Racah, namely finding operators that commute with the observables and whose eigenvalues can be used to break the degeneracy. The paper shows how to find those operators that commute with the $U(N)$ group on an arbitrary $r$-fold tensor product space, and which further generate all such commuting operators. Several examples from $S U(3)$ are given to show how the degenerate spaces are distinguished.

\section{Scattering Operators on Fock Space \\ IV. The Algebra of Operators Commuting \\ with an Internal Symmetry \\ V. The Pseudoscalar Mesons}

W. H. Klink

The goal in both of these papers is to find the algebra of operators that commute with a given symmetry on Fock space. The physical motivation is twofold. On the one hand, we wish to find ways to compute many particle $S$ matrix elements in a nonperturbative way. The phase operator is an element of the algebra of operators commuting with a given symmetry, and it can be used to calculate many particle $S$ matrix elements. 
Second, to fit nuclear energy levels, Iachello uses a Fock space on which the group $U(6)$ arts (corresponding to the $s$ and $d$ bosons) and then looks for chains of subgroups that commute with the angular momentum operators. We want to generalize his procedure and find the algebra of all operators that commute with the angular momentum.

In IV we have solved a slightly simpler problem, namely to construct the algebra of operators that commute with the angular momentum when there are $d$ bosuns only ( $\ell=2$, but not $\ell=0$ bcions). We also show how to construct the representation space for the algebra of commuting operators out of raising and lowering operators.

In $\mathrm{V}$ we construct the algebra of operators that commute with $S U(3)$ on the Fock space generated by the pseudoscalar mesons. The surprising result here is that, although both the group and the Fock spaces in IV and V are very different, the algebra of commuting operators is tive same.

\title{
Elastic Elecîron-Deuteron Scattering
}

\author{
P. L. Chung, W. Pnlyzou, F. Coester, and B. Keister
}

Calculations of elastic electrumagnetic form factors of the deuteron were performed using a front-form [1] formulation of relativistic quantum mechanics. The purpose of these calculations is to understand the structure of the electromagnetic current operator in relativistic quantum meshanical models for momentum transfers relevant to CEBAF and other new electron machines. Knowledge of these operators is essential for the interpretation of the electron-scattering data on larger nuclei.

The result of these calculations was that these models, based on standard nucleonic derrees of freedom, were consistent with all existing data for elastic electron deuteron scattering for momentum transfers between 0 and $8(\mathrm{GeV})^{2}$. This conclusion was made by comparing the result of calculations based on several different nucleon-nucleon interactions 
and several different parameterizations of the single-nucleon form factors with the data. The main source of error in the predictions is due to the experimental uncertainty in the measured neutron electric form factor.

These calculations suggest that the isoscalar contributions to the two-body current for spacelike momentum transfers is well approximated by contributions determined from the single-nucleon currents.

This research and the associated calculations were the major part of Ping Lin Chung's Ph.D. research.

[1] P. L. Chung, F. Coester, B. Keister, and W. N. Polyzou, Phys. Rev. C 37 (1988), 2000 .

\section{Tensor Operators in Poincaré Invariant Models}

W. N. Polyzou and W. H. Klink

The structure of operators that transform covariantly under a unitary representation of the Poincaré group was analyzed [1]. These operators were shown to have a nontrivial interaction dependence. It was shown that this could be understood by applying the Wigner Eckart theorem for the Poincaré group to matrix elements of the desired operator. This permitted a factorization into a Clebsch-Gordan coefficient for the Poincaré group and an invariant reduced matrix element. It was shown that all of the interaction dependence is contained in the Clebsch-Gordan coefficient and the reduced matrix elements are arbitrary invariant functions that correspond to the independent form factors associated with the matrix element. General expression for the invariant form factors were derived and compared to standard expressions for the case of nucleon matrix elements of the electromagnetic current.

[1] W. N. Polyzou and W. H. Klink, Ann. Phys. (NY) 185 (1988), 369. 


\title{
Charge Form Factor of Quark-Model Pions
}

\author{
P. L. Chung, F. Coester, and W. N. Polyzou
}

The charge form factor of the pion was computed in a simple relativistic constituent quark model. The ql: irks were taken to be spin $1 / 2$ particles with form factors appropriate to a structureless point charge. The pion wave function was taken to be a Gaussian in the relative momentum variable. The model had two parameters, namely the rms radius of the Gaussian and the constituent quark masses. The quark masses are involved in the transformation to single quark variables that are needed to compute the current matrix elements. Because this transformation differs from the corresponding nonrelativistic one, the rms radius of the pion wave function and the charge radius cannot be identified.

It was found by varying these two parameters that it is possible to find combinations that fit all low- and high-momentum transfer data. This necessarily ensures agreement with the experimental charge radius which is determined by the temomentum transfer data. In doing this it was found that the nominal rms radius needed to fit these data were about one-half of the experimental charge radius.

No attempt was made to interpret this model in terms of perturbative QCD. There is no justification for using current quark form factors in such a calculation involving constituent quarks; however, the qualitative feature that strong rms radius and charge radius cannot be identified is independent of these considerations. The feature that the strong rms radius is significantly smaller than the charge radius, even for point charges, has important implications in quark models. This is a possible reason for the success of hadronic models of nuclei and needs to be investigated in models of baryons.

[1] P. L. Chung, F. Coester, and W. N. Polyzou, Phys. Lett. B 205 (1988), 54.5. 


\section{Analyzing Multiparticle Reactions I, II}

W. H. Klink

To analyze medium energy production reactions, it is necessary to guarantee that model production amplitudes be properly unitary. One way of doing this is to think of model production amplitudes as elements of partial-wave Hilbert spaces, and calculate the lengths of the model production amplitudes. The length can then be modified in such a way as to satisfy inelastic unitarity. The relationship between properly unitarized partialwave amplitudes and multiplicity and single-particle distribution functions is then worked out.

In II a relativistic Lippmann-Schwinger equation for production reactions is derived. Separable potentials are used to generate exactly soluble models for production amplitudes. These production amplitudes are shown to be elements of the partial-wave Hilbert spaces discussed in I.

\section{Relativistic Two-Body Models \\ W. N. Polyzou}

We have discovered a new technique for adding interactions in relativistic quantum mechanics [1]. The technique is to express a free-particle basis as a direct integral of irreducible representation using Clebsch-Gordan coefficients for the Poincaré group, and then to add interactions in this direct integral representation, which turns out to be a simple linear problem. Different choices of the Clebsch-Gordan coefficients lead to different models.

Dirac's forms of the dynamics are recovered as special cases corresponding to specific choice of Clebsch-Gordan coefficients. The relation of these methods to local field 
theories and manifestly covariant models is discussed. The relation to nonrelativistic scattering phenomenology is also considered.

[1] W. N. Polyzou, Relativistic Two-Body Models, submitted to Ann. Phys. (NY). 


\title{
III. PAPERS PUBLISHED, SUBMITTED OR ACCEPTED
}

\author{
Papers Published \\ (1 January 1988 - 31 December 1988)
}

1. J. Carlson, J. L. Friar, and G. L. Payne

Energy and Symmetry of States in Light Nuclei

Phys. Rev. C 37, 420-422, 1988

2. H. Kröger, R. J. Slobodrian, and G. L. Payne

Direct Calculation of the $S$ Matrix in Coordinate Space

Phys. Rev. C 37, 486-496, 1988

3. J. L. Friar, B. F. Gibson, G. L. Payne, E. L. Tomusiak, and M. Kimura

Trinucieon Magnetic Moments: 34 Channel Results

Phys. Rev. C 37, 2852-2858, 1988

4. J. L. Friar. B. F. Gibson, D. R. Lehman, and G. L. Payne

Trinucleon Asymptotic Normalization Constants: A Comparison of ${ }^{3} \mathrm{He}$ and ${ }^{3} \mathrm{H}$ Phys. Rev. C 37, 2859-2868, 1988

5. J. L. Friar, B. F. Gibson, and G. L. Payne

${ }^{3} \mathrm{H}$ and ${ }^{3} \mathrm{He}$ Solutions for Momentum-Dependent Potentials

Phys. Rev. C 37, 2869-28i2, 1988

6. J. L. Friar and G. L. Payne

Bipolar Harmonic Reduction Formulae

Phys. Rev. C 38, 1-4, 1988

8. G. L. Payne

The Nuclear Three-Body System

Nuclear and Atomic Physics at One Gigaflop

Proceedings Nuclear Science Research Conference Series

[Harwood Academic Publishers, 1988]

9. J. L. Friar, B. F. Gibson, G. L. Pane and S. A. Coon

$S$-Wave $\pi . P$ Scattering Effects in the Nuclear Three-Body Force

Few-Body Systems 5, 13-20, 1988

10. P.E.T. Jorgensen and W. H. Klink

Spectral Transform for the Sub-Laplacian on the Heisenberg Group

Journal d'Analyse . Mathématique 50, 101-121, 1988 
11. W. H. Klink

Scattering Operators on Fock Space

IV. The Algebra of Operators Commuting with an Internal Symmetry

J. Phys. A: Math. Gen. 21, 1988

12. W. H. Klink and T. Ton-That

On Resolving the Multiplicity of Arbitrary Tensor Products

of the $U(N)$ Groups

J. Phys. A: Math. Gen. 21, 3877-3892, 1988

13. W. H. Klink

Scattering Operators on Fock Space

V. The Pseudoscalar Mesons

J. Phys. A: Math. Gen. 21, 4323-4330, 1988

14. W. N. Polyzou and W. H. Klink

The Structure of Poincaré Covariant Tensor Operators in

Quantum Mechanical Models

Ann. Phys. (NY) 185, 369-400, 1988

15. P. L. Chung, F. Coester, D. D. Keister, and W. N. Polyzou

Hamiltonian light-front dynamics of elastic electron-deuteron scattering

Phys. Rev. C 37, 2000-2015, 1988

16. P. L. Chung, F. Coester, and W. N. Polyzou

Charge Form Factors of Quark Model Pions,

Phys. Lett. B 205, 545-552, 1988

\section{Papers Submitted or Accepted}

(as of 31 December 1988)

1. C. R. Chen, G. L. Payne, J. L. Friar, and B. F. Gibson

Low-Energy Nucleon-Deuteron Scattering

Phys. Rev. C [submitted]

2. B. F. Gibson, B. C. Pearce, and G. L. Payne

Triton Model Calculations of the Bonn W-Matrix Rank-One Approximation Phys. Rev. C [submitted]

3. W. H. Klink and T. Ton-That

Decomposition of Holomorphic Hilbert Spaces and $n$-Fold

Tensor Products of $G L(N, \mathcal{C})$

J. Func. Anal. [to be published]

4. W. H. Klink and T. Ton-That

Calculation of Clebsch-Gordan and Racah Coefficients Using

Symbolic Manipulation Programs

J. Comput. Phys. [to be published] 
5. W. H. Klink

The Dual Algebra of the Poincaré Group on Fock Space

Nucl. Phys. [to be published]

6. W. H. Klink

Scattering Operators on Fock Space

VI. The Dual Algebra of the Poincare Group

J. Phys. A: Math. Gen. [submitted]

7. W. H. Klink

Analyzing Multiparticle Reactions:

I. Unitarizing Perturbative Amplitudes

Phys. Rev. C [submitted]

8. W. H. Klink

Analyzing Multiparticle Reactions:

II. Exactly Soluble Production Models

Phys. Rev. C [submitted]

9. W. N. Polyzou

Relativistic Two-Body Models

Ann. Phys. (NY) [submitted] 


\section{Meetings Attended}

G. L. Payne

Computational Atomic and Nuclear Physics at One Gigaflop, Oak Ridge, T.

Spring Meeting of the American Physical Society, Baltimore, MD

W. H. Klink

International Symposium on Spacetime Symmetries, College Park, MD

$17^{\text {th }}$ International Colloquium on Group Theoretical Methods in Physics, Montreal, Canada

W. N. Polyzou

Spring Meeting of the American Physical Society, Baltimore, MD

Continuous Electron Beam Facility Workshop, Newport News, VA

Gordon Research Conference on Photonuclear Physics, Plymouth, NH

Fall Meeting of the American Physical Society, Santa Fe, NM

Midwest Theoretical Physics Workshop, Madison, WI

\section{Invited Talks}

G. L. Payne

"The Nuclear Few-Body System," Computational Atomic and Nuclear Physics at One Gigaflop, Oak Ridge, TN, April 1988

"Low-Energy Proton-Deuteron Scattering," Laval University, Quebec, Canada, June 1988

"Low-Energy p-d Scattering," Argonne National Laboratory, Argonne, IL, November 1988

W. H. Klink

"The Dual Algebra of the Poincaré Group on Fock Space" 
W. N. Polyzou

"Elastic Deuteron Electromagnetic Form Factors," Los Alamos National Laboratory, February 1988

"Relativistic Few-Body Models," Gordon Research Conference on Photonuclear Physics, Plymouth, NH, August 1988 

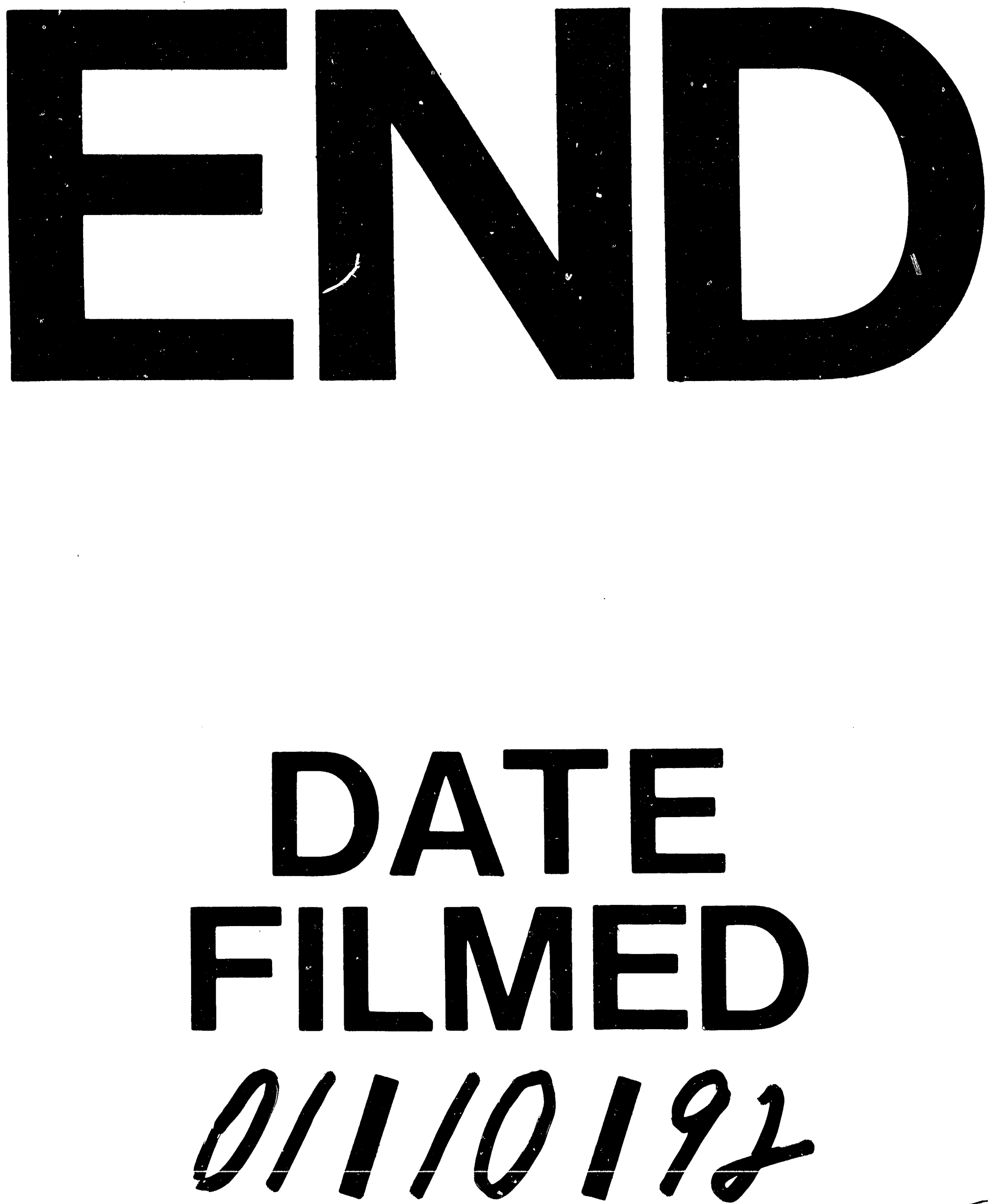
